\title{
Nuclear heavy quark photoproduction in a saturation model
}

\author{
V.P. Gonçalves ${ }^{1}$, M.V.T. Machado ${ }^{1,2}$ \\ 1 Instituto de Física e Matemática, Universidade Federal de Pelotas, Caixa Postal 354, CEP 96010-090, Pelotas, RS, Brazil \\ 2 High Energy Physics Phenomenology Group, GFPAE, IF-UFRGS, Caixa Postal 15051, CEP 91501-970, Porto Alegre, RS, \\ Brazil
}

Received: 6 May 2003 / Revised version: 1 July 2003 /

Published online: 5 September 2003 - (c) Springer-Verlag / Società Italiana di Fisica 2003

\begin{abstract}
We calculate the nuclear inclusive and diffractive cross sections for heavy quark photoproduction within a phenomenological saturation approach. The nuclear cross section is obtained by the extension of the saturation model through the Glauber-Gribov formalism. We predict large nuclear heavy quark cross sections at LHC energies.
\end{abstract}

\section{Introduction}

During the last years the study of high gluon density effects at high energy ( small $x$ ) has become an increasingly active field of research, both from the experimental and the theoretical points of view. In particular, it has been observed that the $e p$ deep inelastic scattering (DIS) data at low $x$ [1] can be successfully described with the help of the saturation model [2], which incorporates the main characteristics of the high density QCD approaches [3-5]. In the high energy regime, the growth of parton distributions should saturate, possibly forming a color glass condensate [5] (for a pedagogical presentation see [6,7]), which is characterized by a bulk momentum scale $Q_{\mathrm{s}}$. If the saturation scale is larger than the QCD scale $\Lambda_{\mathrm{QCD}}$, then this regime can be studied using weak coupling methods. The magnitude of $Q_{\mathrm{s}}$ is associated to the behavior of the gluon distribution at high energies, and some estimates have been obtained. In general, the predictions are $Q_{\mathrm{s}} \sim 1 \mathrm{GeV}$ at HERA/RHIC and $Q_{\mathrm{s}} \sim 2-3 \mathrm{GeV}$ at LHC $[8,9]$.

In the phenomenological analysis presented in $[2,10]$, the critical line which marks the transition to the saturation regime is smaller than $1 \mathrm{GeV}^{2}$, with larger values predicted only for the next generation of $e p$ colliders. However, deep inelastic scattering on nuclei gives us a new possibility to reach a high density QCD phase without requiring extremely low values of $x$. The nucleus in this process serves as an amplifier for the non-linear phenomena expected in QCD at small $x$, obtaining at the accessible energies at HERA and RHIC with an $e A$ collider the parton densities which would be probed only at energies comparable to LHC energies with an ep collider. In order to understand this expectation and estimate the kinematic region where the high densities effects should be present, we can analyze the behavior of the function $\kappa\left(x, Q^{2}\right) \equiv \frac{3 \pi^{2} \alpha_{\mathrm{s}}}{2 Q^{2}} \frac{x g_{A}\left(x, Q^{2}\right)}{\pi R_{A}^{2}}$, which represents the probability of a gluon-gluon interaction inside the parton cascade, and also is denoted the packing factor of partons in a parton cascade $[4,11]$. Considering that the condition $\kappa=1$ specifies the critical line, which separates the linear (low parton density) regime $\kappa \ll 1$ and the high density regime $\kappa \gg 1$, we can define the saturation momentum scale $Q_{\mathrm{s}}$ given by ${ }^{1}$

$$
Q_{\mathrm{s}}^{2}(x ; A)=\frac{3 \pi^{2} \alpha_{\mathrm{s}}}{2} \frac{x g_{A}\left(x, Q_{\mathrm{s}}^{2}(x ; A)\right)}{\pi R_{A}^{2}},
$$

below which the gluon density reaches its maximum value (saturates). At any value of $x$ there is a value of $Q^{2}=$ $Q_{\mathrm{s}}^{2}(x)$ in which the gluonic density reaches a sufficiently high value that the number of partons stops to rise. This scale depends on the energy of the process $\left[x g \propto x^{-\lambda}\right.$ $(\lambda \approx 0.3)]$ and on the atomic number of the colliding nuclei $\left[R_{A} \propto A^{\frac{1}{3}} \rightarrow Q_{\mathrm{s}}^{2} \propto A^{\frac{1}{3}}\right]$, with the saturation scale for nuclear targets larger than for the nucleon ones. Therefore, we expect that the saturation effects should be manifest in nuclear collisions. Recently, a phenomenological analysis of the multiplicity distributions of particles produced in heavy ion collisions at RHIC, considering the saturation of the nuclear wavefunction, reproduced the experimental data very well [14]. However, the current situation is not unambiguous, since other approaches which do not assume saturation describe the same set of data. This result motivates more extensive studies of nuclear collisions and, in particular, of electron-nucleus collisions at high energies, where the number of other medium effects is reduced in comparison with $A A$ collisions. Recently, some authors $[13,15,16]$ have addressed this subject with particular emphasis on the behavior of the nuclear structure

\footnotetext{
1 No exact definition of the saturation scale is known so far. In particular, (1) is valid in the double logarithmic approximation. A more detailed analysis would imply that the enhancement between the nucleon and nuclear cases is smaller than the one predicted in this approximation (see e.g. $[12,13]$ ).
} 
functions (for previous studies see e.g. [4,17-19]), obtaining predictions which agree with the scarce experimental data. Since these models rely on different assumptions, more accurate experimental results for the nuclear structure function in a large kinematical region are necessary to probe the high density dynamics. However, these results compel us to suggest further measurements in electronnuclei interactions.

Our goal in this paper is to investigate the high energy heavy quark photoproduction on nuclei targets using the saturation hypothesis. Nuclear photoproduction has recently been studied in [20], obtaining a quite reasonable description of the experimental data, but the specific topic of heavy quark production was not addressed in that analysis. It is important to emphasize that for heavy quark production the predictions are not dependent on the hypothesis for the soft region (e.g. the values of the light quark masses). Here we study the nuclear photoproduction of heavy quarks considering the approach proposed in [13], which is simpler, gives an equally reasonable agreement with nuclear data as other approaches, and is associated with a model which gives a good description of inclusive and diffractive $e p$ experimental data. A detailed comparison between the distinct saturation approaches for the nuclear case will be presented in a separate publication [21].

This paper is organized as follows. In the next section we present a brief review of the saturation model for the nucleon and nuclear case, and the dipole nuclear cross section is analyzed. Section 3 presents our predictions for the energy and atomic number dependence of the heavy quark photoproduction cross section, as well as a detailed analysis of the overlap functions, which allow us to verify what the mean dipole size is that contributes for this process. As a by-product, in Sect. 4 the diffractive production of heavy quarks in photonuclear collisions is also considered in the saturation model. Finally, in Sect. 5 we summarize our conclusions.

\section{Nuclear cross sections in a saturation model}

In this section we shortly review the main concepts and formulae of the saturation model which is based on the color dipole approach. The latter gives a simple unified picture of inclusive and diffractive processes which provides a large phenomenology in the DIS regime. In this approach, the scattering process can be seen in the target rest frame as a succession in time of three factorizable subprocesses:

(i) the photon fluctuates in a quark-antiquark pair,

(ii) this color dipole interacts with the target, and

(iii) the quark pair annihilates in a virtual photon.

Using as kinematic variables the $\gamma^{*} N$ CMS energy squared $s=W^{2}=(p+q)^{2}$, where $p$ and $q$ are the target and the photon momenta, respectively, the photon virtuality squared $Q^{2}=-q^{2}$ and the Bjorken variable $x=Q^{2} /\left(W^{2}+Q^{2}\right)$, the corresponding total cross section reads $[2,22]$

$$
\begin{aligned}
& \sigma_{\mathrm{T}, \mathrm{L}}\left(x, Q^{2}\right) \\
& =\int_{0}^{1} \mathrm{~d} z \int \mathrm{d}^{2} \boldsymbol{r}\left|\Psi_{\mathrm{T}, \mathrm{L}}\left(z, \boldsymbol{r}, Q^{2}\right)\right|^{2} \sigma_{\operatorname{dip}}^{\mathrm{target}}\left(\tilde{x}, \boldsymbol{r}^{2}\right),
\end{aligned}
$$

where

$$
\begin{aligned}
& \left|\Psi_{\mathrm{T}}\left(z, \boldsymbol{r}, Q^{2}\right)\right|^{2}=\frac{6 \alpha_{\mathrm{em}}}{4 \pi^{2}} \\
& \times \sum_{f} e_{f}^{2}\left\{\left[z^{2}+(1-z)^{2}\right] \varepsilon^{2} K_{1}^{2}(\varepsilon r)+m_{f}^{2} K_{0}^{2}(\varepsilon r)\right\} \\
& \left|\Psi_{\mathrm{L}}\left(z, \boldsymbol{r}, Q^{2}\right)\right|^{2} \\
& =\frac{6 \alpha_{\mathrm{em}}}{\pi^{2}} \sum_{f} e_{f}^{2}\left\{Q^{2} z^{2}(1-z)^{2} K_{0}^{2}(\varepsilon r)\right\}
\end{aligned}
$$

are the squared photon wavefunction for transverse $(\mathrm{T})$ and longitudinal (L) photons, respectively. The variable $\boldsymbol{r}$ defines the relative transverse separation of the pair (dipole) and $z(1-z)$ is the longitudinal momentum fraction of the quark (antiquark). The auxiliary variable $\varepsilon^{2}=$ $z(1-z) Q^{2}+m_{f}^{2}$ depends on the quark mass, $m_{f}$. The $K_{0,1}$ are the McDonald functions and the summation is performed over the quark flavors.

For electron-proton interactions, the dipole cross section $\sigma_{\text {dip }}^{p}$, describing the dipole-proton interaction is substantially affected by non-perturbative content. There are several phenomenological implementations for this quantity [23]. The main feature of these approaches is to be able to match the soft (low $Q^{2}$ ) and hard (large $Q^{2}$ ) regimes in an unified way. In the present work we follow the quite successful saturation model [2], which interpolates between the small and large dipole configurations, providing color transparency behavior, $\sigma_{\text {dip }} \sim \boldsymbol{r}^{2}$, as $\boldsymbol{r} \rightarrow 0$ and constant behavior, $\sigma_{\text {dip }} \sim \sigma_{0}$, at large dipole separations. The parameters of the model have been obtained from an adjustment to small $x$ HERA data. Its parameter-free application to diffractive DIS has been also quite successful [2] as well as its extension to virtual Compton scattering [24], vector meson production [25] and two-photon collisions [26]. The parameterization for the dipole cross section takes the eikonal-like form,

$$
\begin{aligned}
\sigma_{\text {dip }}^{p}\left(\tilde{x}, \boldsymbol{r}^{2}\right) & =\sigma_{0}\left[1-\exp \left(-\frac{Q_{\mathrm{s}}^{2}(x) \boldsymbol{r}^{2}}{4}\right)\right], \\
Q_{\mathrm{s}}^{2}(x) & =\left(\frac{x_{0}}{\tilde{x}}\right)^{\lambda} \mathrm{GeV}^{2}
\end{aligned}
$$

where the saturation scale $Q_{\mathrm{s}}^{2}$ defines the onset of the saturation phenomenon, which depends on energy. The parameters were obtained from a fit to the HERA data producing $\sigma_{0}=23.03(29.12) \mathrm{mb}, \lambda=0.288(0.277)$ and $x_{0}=3.04 \cdot 10^{-4}\left(0.41 \cdot 10^{-4}\right)$ for a three-flavor (four-flavor) analysis [2] (see $[10,16]$ for improvements of this model). An additional parameter is the effective light quark mass, $m_{f}=0.14 \mathrm{GeV}$, consistent with the pion mass. It should 

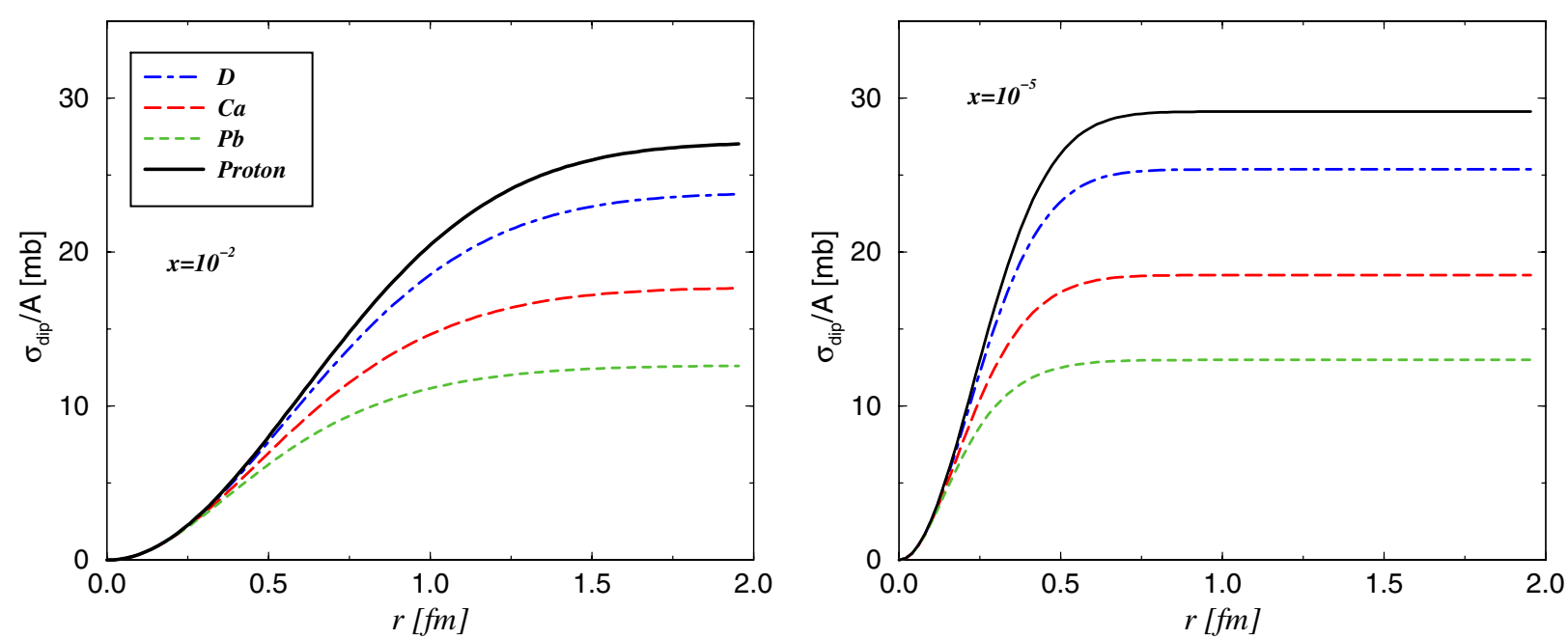

Fig. 1. The nuclear dipole cross section as a function of dipole size $r$ for different $x$ values and distinct $A$, including the proton case. The results are normalized to $A$

be noticed that the quark mass plays the role of a regulator for the photoproduction $\left(Q^{2}=0\right)$ cross section. The light quark mass is one of the non-perturbative inputs in the model. The charm quark mass is considered to be $m_{c}=$ $1.5 \mathrm{GeV}$. A smooth transition to the photoproduction limit is obtained with a modification of the Bjorken variable:

$$
\tilde{x}=x\left(1+\frac{4 m_{f}^{2}}{Q^{2}}\right)=\frac{Q^{2}+4 m_{f}^{2}}{W^{2}} .
$$

The saturation model is suitable in the region below $x=$ 0.01 and the large $x$ limit still needs a consistent treatment. Making use of the dimensional-cutting rules, here we supplement the dipole cross section, (5), with a threshold factor $(1-x)^{n_{\text {thres }}}$, taking $n_{\text {thres }}=5$ for a three-flavor analysis and $n_{\text {thres }}=7$ for a four-flavor one. This procedure ensures a consistent description of heavy quark production at the fixed target data [27].

Before going further, some comments on the impact parameter dependence of the saturation model are of concern to us. The implicit assumption in the approach is that the proton is treated as being homogeneous in the transverse plane. In such a case, the impact parameter profile is given by the Heaviside function, $S(b)=\Theta\left(b_{0}-b\right)$. The profile is considered to be peaked at central impact parameter, namely at $b=0$. Actually, this procedure is oversimplified and more realistic profiles can be considered. For phenomenological purposes a gaussian or a hard sphere assumption are commonly taken into account. Recently, the impact parameter dipole saturation model [16] was developed, recovering the known Glauber-Mueller dipole cross section. There, distinct shapes for $S(b)$ were considered and their parameters constrained from data on the $t$-dependence of the $J / \Psi$ photoproduction.

Let us discuss the extension of the saturation model for the photon-nucleus interactions. Here, we follow the simple procedure proposed in [13], which consists of an extension to nuclei, using the Glauber-Gribov picture [28], of the saturation model discussed above, without any new pa- rameter. (For similar approaches see e.g. [4,29].) There, the nuclear version is obtained replacing the dipole-nucleon cross section in (2) by the nuclear one,

$$
\begin{aligned}
& \sigma_{\text {dip }}^{A}\left(\tilde{x}, r^{2}, A\right) \\
& =\int \mathrm{d}^{2} b 2\left\{1-\exp \left[-\frac{1}{2} A T_{A}(b) \sigma_{\text {dip }}^{p}\left(\tilde{x}, r^{2}\right)\right]\right\},
\end{aligned}
$$

where $b$ is the impact parameter of the center of the dipole relative to the center of the nucleus, and the integrand gives the total dipole-nucleus cross section for a fixed impact parameter. The nuclear profile function is labelled by $T_{A}(b)$, which will be obtained from a three-parameter Fermi distribution for the nuclear density [30]. The above equation sums up all the multiple elastic rescattering diagrams of the $q \bar{q}$ pair and is justified for a large coherence length, where the transverse separation $r$ of the partons in the multiparton Fock state of the photon becomes a quantity as well conserved as the angular momentum, i.e. the size of the pair's $r$ becomes the eigenvalue of the scattering matrix. It is important to emphasize that for very small values of $x$, other diagrams beyond the multiple pomeron exchange considered here should contribute (e.g. pomeron loops) and a more general approach for the high density (saturation) regime must be considered. However, we believe that this approach allows us to obtain lower limits of the high density effects in the RHIC and LHC kinematic range. Therefore, at first glance, the region of applicability of this model should be at small values of $x$, i.e. large coherence lengths, and for not too high values of virtualities, where the implementation of the DGLAP evolution should be required. Therefore, the approach is quite suitable for the analysis of heavy quark photoproduction in the RHIC and LHC kinematical ranges.

In order to investigate the dependence of the nuclear dipole cross section on the dipole size, in Fig. 1 are shown our results for $\sigma_{\text {dip }}^{A}$ as a function of the dipole size at two fixed values of $x$. We have selected the different nuclei $A=$ $\mathrm{D}, \mathrm{Ca}$ and $\mathrm{Pb}$ as well the proton case at $x=10^{-2}$ and 

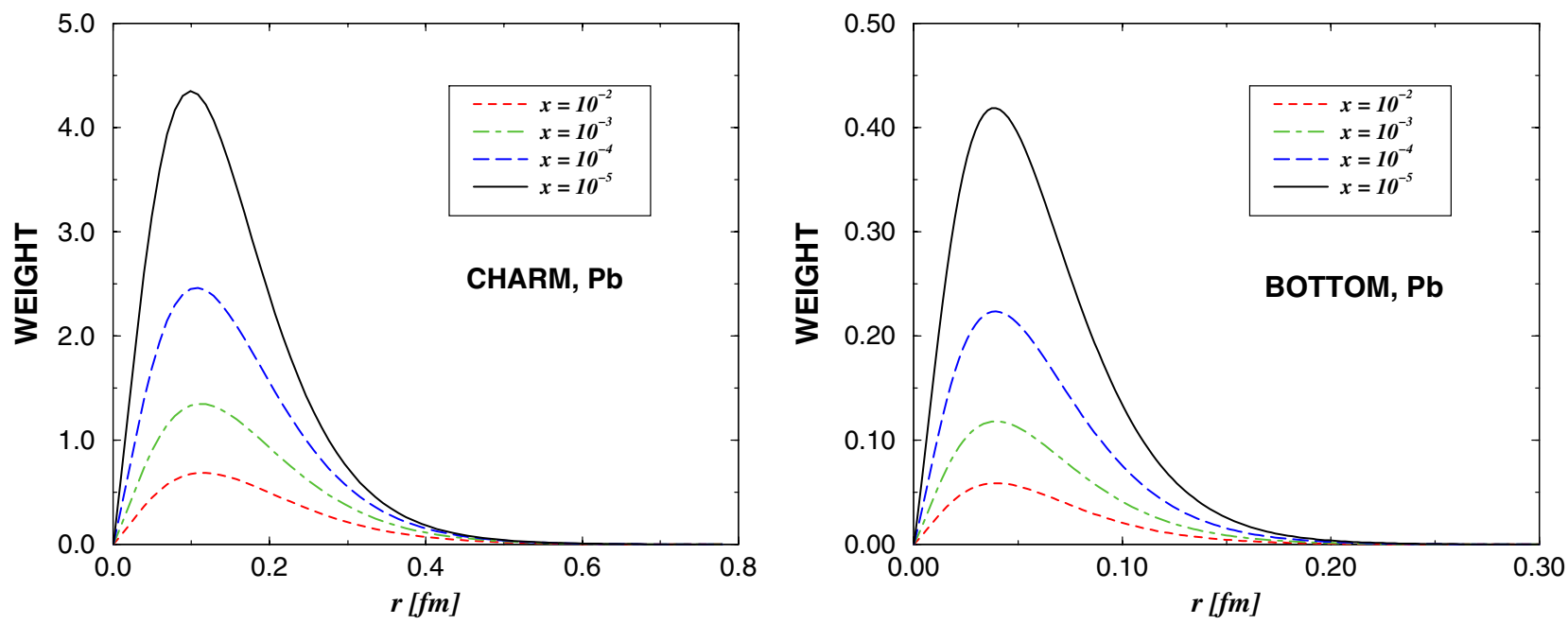

Fig. 2. The $r$-dependence of the photon-nucleus overlap function for charm and bottom production, different $x$ and $A=\mathrm{Pb}$

$x=10^{-5}$, with the dipole cross section normalized to $A$. The dependence of the behavior on $r$ is the same as in the proton case, saturating at large values of $r$. At small dipole sizes the result is almost independent of $A$, whereas at large dipole sizes the nuclear suppression is quite substantial and dependent on $A$. For instance, the reduction in the overall normalization reaches a factor of about 3 for lead in comparison with the nucleon case at small $x$. This result has sizable consequences for processes where the large dipole contribution is important, for example in the transversal component of $F_{2}^{A}$ and diffractive production of light quarks. For heavy quarks, the situation is different since the corresponding wavefunctions select mostly small dipoles configurations, as we will show later on. Having addressed the main concepts and definitions, in the next section we present our results for the heavy quark production within the color dipole picture supplemented by the saturation model discussed above. Here, we will focus on photoproduction of charm and bottom in the kinematical range relevant to RHIC and LHC.

\section{Nuclear heavy quark photoproduction}

In this section we compute the nuclear cross section for the photoproduction of charm and bottom quarks. In this case the non-perturbative input associated with the light quark mass is avoided and we are left with the hard scale given by the heavy quark masses. Here, the following values are taken into account: $m_{c}=1.5 \mathrm{GeV}$ and $m_{b}=4.5 \mathrm{GeV}$, in order to be consistent with the previous analysis using the saturation model in the nucleon case. Notice that the use of a different choice implies a distinct overall normalization in the final result.

The cross section for heavy quark photoproduction on nuclei targets is given by

$$
\begin{aligned}
& \sigma_{\text {tot }}^{\gamma A}(W, A) \\
& =\int_{0}^{1} \mathrm{~d} z \int \mathrm{d}^{2} \boldsymbol{r}\left|\Psi_{\mathrm{T}}\left(z, \boldsymbol{r}, Q^{2}=0\right)\right|^{2} \sigma_{\mathrm{dip}}^{A}\left(\tilde{x}, \boldsymbol{r}^{2}, A\right),
\end{aligned}
$$

where the longitudinal contribution is suppressed [see (4) at $\left.Q^{2}=0\right]$ and $e_{c, b}^{2}=4 / 9$ and $1 / 9$, respectively. Accordingly, $m_{f}=m_{c, b}$ in (3) and the parameters for the dipole cross section are taken from the four-flavor analysis. The formula (9) sums up in a compact form all the elastic + inelastic rescattering diagrams of the heavy quark pair with the nucleus.

Before presenting our results for the energy dependence of the cross section, we can investigate the mean dipole size dominating the nuclear heavy quark photoproduction. We define the photon-nucleus overlap function which reads

$$
\mathcal{W}(\tilde{x}, \boldsymbol{r}, A)=2 \pi r \int \mathrm{d} z\left|\Psi_{\mathrm{T}}(z, \boldsymbol{r})\right|^{2} \sigma_{\mathrm{dip}}^{A}\left(\tilde{x}, \boldsymbol{r}^{2}, A\right) \text {. }
$$

In Fig. 2 are shown the overlap function for the charm and bottom production as a function of the dipole size. They are computed for the lead nucleus and for different $x$. In the charm case, the distribution is peaked at approximately $r=0.1 \mathrm{fm}$, whereas for the bottom case this value is shifted to $r=0.05 \mathrm{fm}$, which agrees with the theoretical expectation that the $q \bar{q}$ pairs have a typical transverse size $\approx 1 / m_{f}[29]$. Therefore, the main contribution to the cross section comes from the small dipole sizes, i.e. from the perturbative regime. In contrast, for light quarks a broader $r$ distribution is obtained, peaked for large values of the pair separation, implying that non-perturbative contributions cannot be disregarded in that case. For the sake of illustration, the transition region between perturbative and non-perturbative regimes ranges at $Q^{2} \sim 1 / r^{2} \simeq 1 \mathrm{GeV}^{2}$, which means a perturbative domain for $r \leq 0.2-0.4 \mathrm{fm}$. Concerning the high density effects, its value is more sizable at large dipole configurations (see Fig. 1), meaning that for heavy quark photoproduction we should expect that the associated modifications will be small.

In Fig. 3 are shown the results for the charm and bottom photoproduction cross section as a function of energy for different nuclei, including the proton case. The results present a mild growth on $W$ at high energies stemming from the saturation model, whereas the low energy region is consistently described through the threshold factor. For 

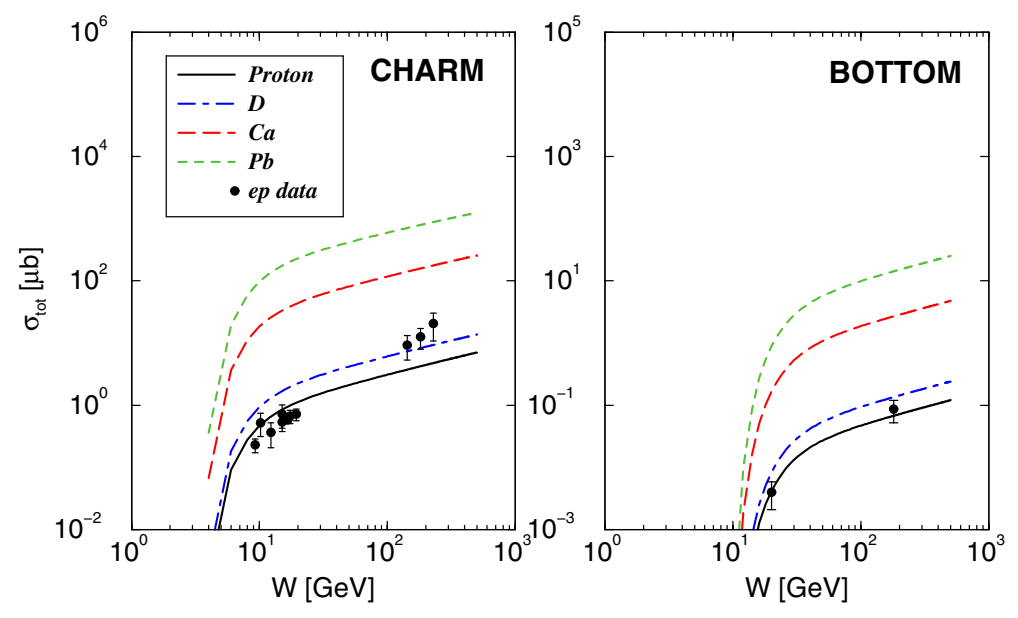

Fig. 3. The total nuclear cross section for charm and bottom photoproduction as a function of CMS energy $W$ and distinct nuclei the proton, the experimental data from HERA [31] and fixed target collisions [32] are also included for the sake of comparison. The result for charm underestimates the data by a factor 2 at $W_{\gamma p} \simeq 200 \mathrm{GeV}$, whereas it is consistent with the measurements of the bottom cross section. Concerning charm production, the measured cross sections present a well-known steeper behavior in dependence on energy even at electroproduction, suggesting that further resummations in the original saturation model are needed in order to produce the larger growth with energy appearing in the charm measurements. We believe that the better result for bottom happens to be a mismatch between a large uncertainty in the experimental measurement and the lower bottom mass $m_{b}=4.5 \mathrm{GeV}$ considered here. For the nuclear case, we predict that their absolute values are rather large, reaching $\approx 2 \cdot 10^{3}$ and $\approx 40 \mu \mathrm{b}$ for charm and bottom for lead at $W=10^{3} \mathrm{GeV}$. It is important to emphasize that our results agree with the predictions obtained for charm photoproduction in [33], where the heavy flavor leptoproduction off the nucleus has been addressed considering an approach which resums the fan diagrams of BFKL pomerons, with initial condition for the evolution at $x=0.01$ given by (5). For bottom photoproduction, our results are smaller than in [33]. We believe that our results are reliable, since they are consistent with the simple expectation $\sigma_{\gamma A \rightarrow b \bar{b} X} \approx A \times \sigma_{\gamma p \rightarrow b \bar{b} X}$ for the color transparency regime. A check of the saturation model for the proton case can be found in [27] and a comparison with other approaches in [21]. As expected from the discussion in the previous paragraph, the nuclear cross sections can be reasonably approximated by $A$ times the nucleon cross section (color transparency) [34], since this process is dominated by the scattering of small size dipoles. This result is consistent with the behavior of the dipole cross section presented in Fig. 1, where we have seen that sizable nuclear effects are only important at large dipole sizes.

Here, an additional issue should be addressed. The dipole-nucleon cross section resums higher twist contributions and also ensures the unitarity requirements, producing a constant value at large $r$. In order to investigate the effects of this resummation in the dipole-nucleus cross section, we show in Fig. 4a our results for the nuclear pho- toproduction cross section considering as input the full dipole-nucleon cross section (solid lines), see (5), and only the leading term in the expansion of $\sigma_{\text {dip }}^{p}$ (dot-dashed lines). They were computed for charm quark and considering the calcium and lead nuclei. The effect is almost negligible for a light nucleus, whereas it is more sizable for heavy ones at high energies. This result demonstrates that nuclear heavy quark production is not sensitive to the assumption of a saturated nucleon. In contrast, the behavior of the nuclear structure function depends on the saturation effects at the nucleon level, as shown in [19].

\section{Diffractive photoproduction of heavy quarks}

Let us now compute the diffrative production of heavy quarks. This process was analyzed for $e p$ collisions in [3537]. In terms of the $S$-matrix at a given impact parameter of the collision, $S(b)$, the total and elastic cross sections for the deep inelastic scattering on a nucleus are given by [38]

$$
\begin{aligned}
\sigma_{\text {tot }} & =2 \int \mathrm{d}^{2} b[1-S(b)], \\
\sigma_{\text {el }} & =\int \mathrm{d}^{2} b[1-S(b)]^{2},
\end{aligned}
$$

which demonstrate how easily the elastic and total cross sections could be obtained from one another. Identifying $1-S(b)$ with the expression within the brackets in (8) and considering that diffraction of the photon on the target can be thought as an elastic scattering of each dipole off the nucleus, we obtain for the nuclear diffractive cross section

$$
\begin{aligned}
\sigma_{\mathrm{T}, \mathrm{L}}^{\text {Diff }}\left(x, Q^{2}\right) \\
=\int \mathrm{d}^{2} b \int_{0}^{1} \mathrm{~d} z \int \mathrm{d}^{2} \boldsymbol{r}\left|\Psi_{\mathrm{T}, \mathrm{L}}\left(z, \boldsymbol{r}, Q^{2}\right)\right|^{2} \\
\quad \times\left\{1-\exp \left[-\frac{1}{2} A T_{A}(b) \sigma_{\text {dip }}^{p}\left(\tilde{x}, \boldsymbol{r}^{2}\right)\right]\right\}^{2} .
\end{aligned}
$$

For heavy quark photoproduction we see that only the transverse polarization contributes and the wavefunction is dependent on the heavy quark masses. 


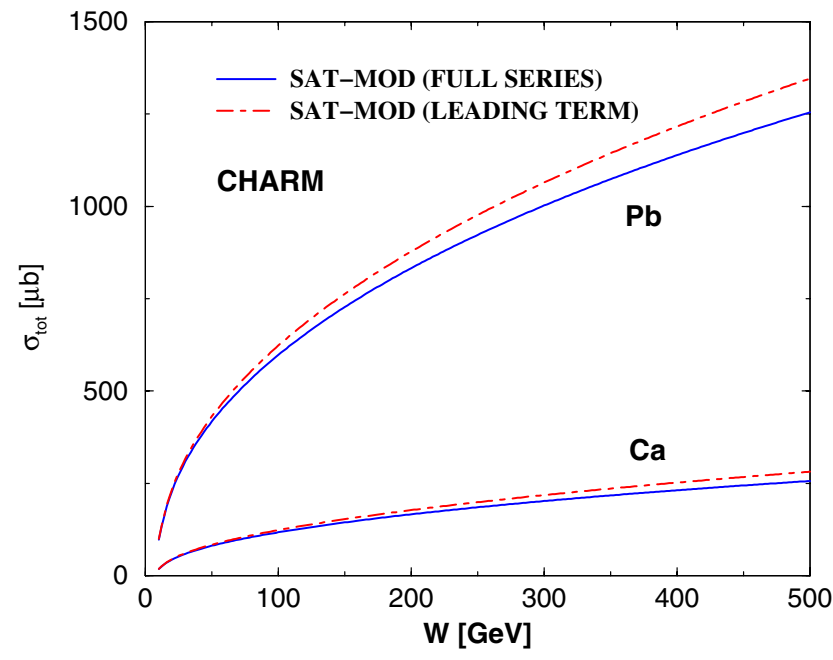

a

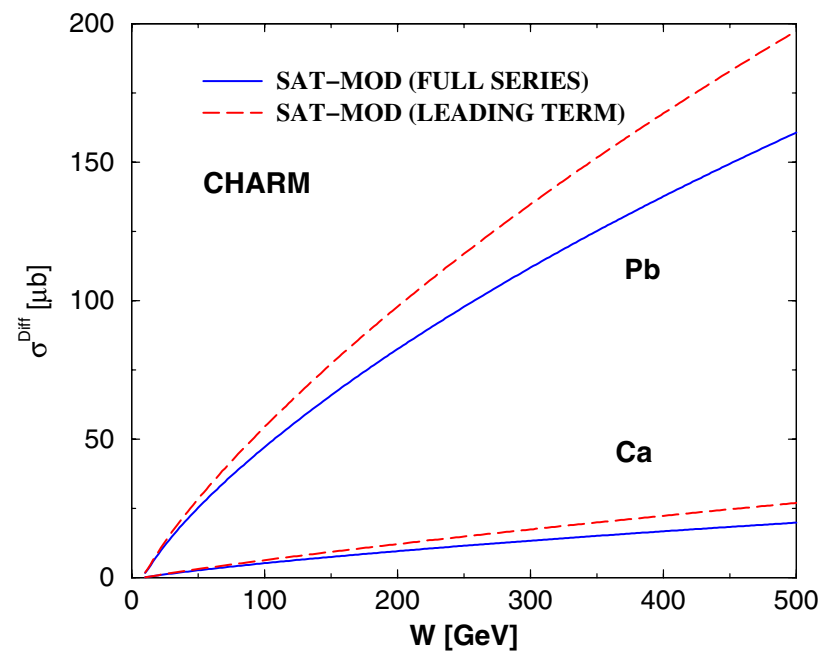

b

Fig. 4a,b. The result for the a inclusive and $\mathbf{b}$ diffractive nuclear charm photoproduction cross section as a function of CMS energy for calcium and lead. The solid line corresponds to the full dipole nucleon cross section of the saturation model (full series) and the dot-dashed line is obtained using only the leading term
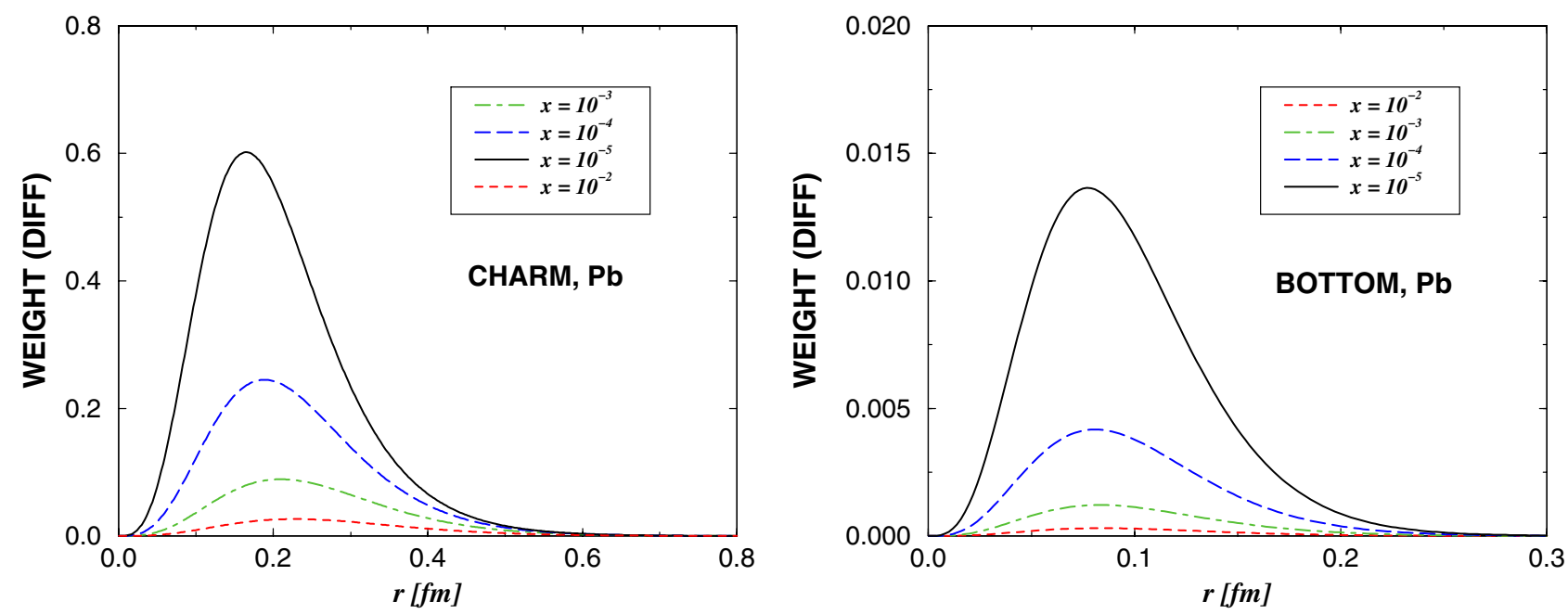

Fig. 5. The $r$-dependence of the diffractive photon-nucleus overlap function for charm and bottom production, different $x$ and $A=\mathrm{Pb}$

A similar analysis of the mean size dipole dominance can be made for the diffractive case. Introducing the diffractive overlap function defined by

$$
\begin{aligned}
& \mathcal{W}^{\text {Diff }}(\tilde{x}, \boldsymbol{r}, A) \\
& =2 \pi r \int \mathrm{d}^{2} b \int \mathrm{d} z\left|\Psi_{\mathrm{T}}(z, \boldsymbol{r})\right|^{2} \\
& \quad \times\left\{1-\exp \left[-\frac{1}{2} A T_{A}(b) \sigma_{\mathrm{dip}}^{p}\left(\tilde{x}, \boldsymbol{r}^{2}\right)\right]\right\}^{2},
\end{aligned}
$$

in Fig. 5 we present our results for the $r$-dependence of this function for different values of $x$ and $A=\mathrm{Pb}$. We can see that the distribution is broader and its maximum is shifted to large values of $r$ in comparison with the inclusive case. This result is expected since the diffractive production has a large amount of non-perturbative contributions. However, similarly to the inclusive case, the mean size dipole occurs in the perturbative regime, with the large distance still strongly suppressed.

In Fig. 6 the results are shown for the charm and bottom diffractive cross section as a function of energy for different nuclei. The values at high energies are sizable, reaching $250 \mu \mathrm{b}$ and $0.8 \mu \mathrm{b}$ for charm and bottom production, considering lead at $W=10^{3} \mathrm{GeV}$. These values are in agreement with a $10 \%$ contribution from diffractive scattering to the total cross section observed in photonnucleon reactions. Concerning the dependence on $A$, the results present an approximate behavior $\sigma^{\text {Diff }} \simeq A^{4 / 3}$, as expected for the interaction of small dipoles with the nucleus $[39,40]$. We also have checked the diffractive production of heavy quarks for the proton case, with a cross section of order $100 \mathrm{nb}$ at $W=200 \mathrm{GeV}$ for diffractive open charm production, being consistent with previous theoretical estimates [37]. There, a value of $60 \mathrm{nb}$ was found 


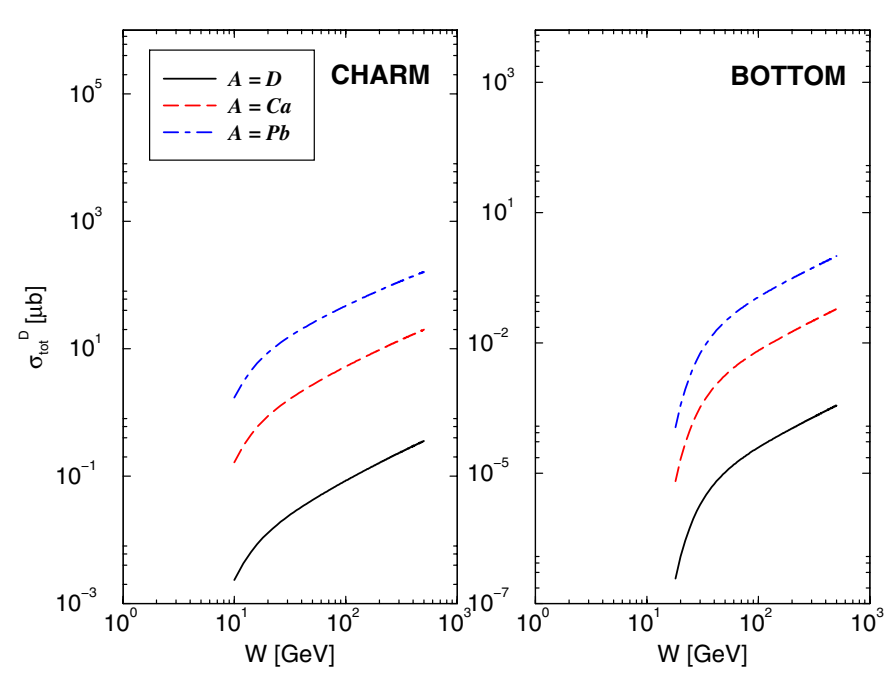

Fig. 6. The diffractive nuclear cross section for charm and bottom photoproduction as a function of CMS energy $W$ and for distinct nuclei

at the same energy in the photoproduction case. A more detailed analysis for the proton case should be addressed elsewhere.

Finally, in Fig. 4b we analyze the dependence of the diffractive nuclear cross section on considering or not a saturated proton, namely saturation effects in the nucleon level. We can see that while for light nuclei the cross section is not sensitive to the assumption of a saturated nucleon; for heavy nuclei the result differs by about $20 \%$. Therefore, the analysis of the diffractive cross section can be useful to constrain the nucleon dynamics.

\section{Summary and conclusions}

In this paper we have calculated the nuclear inclusive and diffractive cross sections for heavy quark photoproduction within a phenomenological saturation model. In such a model the nuclear cross section is obtained through the Glauber-Gribov formalism. Since it describes reasonably the experimental data for the nuclear structure function, we are confident in extending this model for the nuclear heavy quark photoproduction case. Moreover, it is simple and relies on a model which gives a good description of inclusive and diffractive ep experimental data. This model should be valid until the full non-linear evolution effects become important, which implies the consideration of the pomeron loops beyond the multiple scattering on single nucleons estimated in the present framework. We have verified that the main contribution of the high density effects occur for large pair separation, while for dipoles of small size they are almost negligible. We have investigated the mean dipole size dominance and have verified that the heavy quark cross section is dominated by a small dipole configuration, in contrast to the light quark case. Consequently, we have the result that the nuclear heavy quark production is not strongly modified by the high density effects. We predict the absolute values for the cross section to be rather large, being about $2 \mathrm{mb}$ and $0.04 \mathrm{mb}$ for charm and bottom, respectively, for lead and $W=1 \mathrm{TeV}$. These values are similar to those resulting from the resummation of the fan diagrams of BFKL pomerons. Furthermore, we have computed the diffractive nuclear photoproduction of heavy quarks. This quantity is more sensitive to the nonperturbative sector, corresponding to larger dipole sizes. This means that the cross section is dominated by larger dipole configurations than in the inclusive case. The results take values about $250 \mu \mathrm{b}$ and $0.8 \mu \mathrm{b}$ for charm and bottom for lead at $W=1 \mathrm{TeV}$. Concerning the $A$-dependence, we have found a behavior proportional to $A$ and $A^{4 / 3}$ for the inclusive and diffractive cross sections, respectively, in agreement with theoretical expectations associated with the color transparency regime.

Acknowledgements. M.V.T.M. thanks for their support the High Energy Physics Phenomenology Group at the Institute of Physics, GFPAE IF-UFRGS, Porto Alegre. This work was partially financed by the Brazilian funding agencies $\mathrm{CNPq}$ and FAPERGS.

\section{References}

1. M. Klein, Int. J. Mod. Phys. A 15S1, 467 (2000)

2. K. Golec-Biernat, M. Wüsthoff, Phys. Rev. D 59, 014017 (1999); Phys. Rev. D 60, 114023 (1999)

3. Ia. Balitsky, Nucl. Phys. B 463, 99 (1996); Yu. Kovchegov, Phys. Rev. D 60, 034008 (1999)

4. A.L. Ayala, M.B. Gay Ducati, E.M. Levin, Nucl. Phys. B 493, 305 (1997)

5. E. Iancu, A. Leonidov, L. McLerran, Nucl. Phys. A 692, 583 (2001); E. Ferreiro, E. Iancu, A. Leonidov, L. McLerran, Nucl. Phys. A 703, 489 (2002)

6. E. Iancu, A. Leonidov, L. McLerran, hep-ph/0202270

7. E. Iancu, R. Venugopalan, hep-ph/0303204

8. M. Gyulassy, L. McLerran, Phys. Rev. C 56, 2219 (1997)

9. V.P. Gonçalves, Phys. Lett. B 495, 303 (2000)

10. J. Bartels, K. Golec-Biernat, H. Kowalski, Phys. Rev. D 66, 014001 (2002)

11. L.V. Gribov, E.M. Levin, M.G. Ryskin, Phys. Rept. 100, 1 (1983)

12. E. Levin, K. Tuchin, Nucl. Phys. A 693, 787 (2001); E. Levin, M. Lublinsky, Nucl. Phys. A 696, 833 (2001)

13. N. Armesto, Eur. Phys. J. C 26, 35 (2002)

14. D. Kharzeev, M. Nardi, Phys. Lett. B 507, 121 (2001); D. Kharzeev, E. Levin, Phys. Lett. B 523, 79 (2001)

15. N. Armesto et al., hep-ph/0304119

16. H. Kowalski, D. Teaney, hep-ph/0304189

17. K.J. Eskola, J.W. Qiu, X.N. Wang, Phys. Rev. Lett. 72, 36 (1994)

18. Z. Huang, H.J. Lu, I. Sarcevic, Nucl. Phys. A 637, 79 (1998)

19. M.B. Gay Ducati, V.P. Gonçalves, Phys. Rev. C 60, 058201 (1999)

20. J. Bartels et al., hep-ph/0304166

21. V.P. Gonçalves, M.V.T. Machado, hep-ph/0307129

22. N.N. Nikolaev, B.G. Zakharov, Phys. Lett. B 332, 184 (1994); Z. Phys. C 64, 631 (1994) 
23. J.R. Forshaw, G. Kerley, G. Shaw, Phys. Rev. D 60, 074012 (1999); M. McDermott, L. Frankfurt, V. Guzey, M. Strikman, Eur. Phys. J. C 16, 641 (2000); E. Gotsman et al., J. Phys. G 27, 2297 (2001); A. Donnachie, H.G. Dosch, Phys. Rev. D 65, 014019 (2002); M.B. Gay Ducati, M.V.T. Machado, Phys. Rev. D 65, 114019 (2002); M.A. Betemps, M.B. Gay Ducati, M.V.T. Machado, Phys. Rev. D 66, 014018 (2002)

24. L. Favart, M.V.T. Machado, Eur. Phys. J. C 29, 365 (2003)

25. A.C. Caldwell, M.S. Soares, Nucl. Phys. A 696, 125 (2001)

26. N. Timneanu, J. Kwiecinski, L. Motyka, Eur. Phys. J. C 23, 513 (2002)

27. C. Brenner Mariotto, M.B. Gay Ducati, M.V.T. Machado, Phys. Rev. D 66, 114013 (2002)

28. V.N. Gribov, Sov. Phys. JETP 29, 483 (1969); Sov. Phys. JETP 30, 709 (1970)

29. N.N. Nikolaev, B.G. Zakharov, Phys. Lett. B 260, 414 (1991); Z. Phys. C 49, 607 (1991)

30. C.W. De Jager, H. De Vries, C. De Vries, Atom. Data Nucl. Data Tabl. 14, 479 (1974)

31. S. Aid et al. (H1 collaboration), Nucl. Phys. B 472, 32 (1996); C. Adloff et al. (H1 collaboration), Phys. Lett. B 467, 156 (1999)
32. M.S. Atiya et al., Phys. Rev. Lett. 43, 414 (1979); D. Aston et al. (WA4 collaboration), Phys. Lett. B 94, 113 (1980); J.J. Aubert et al. (EMC collaboration), Nucl. Phys. B 213, 31 (1983); K. Abe et al. (SHFP collaboration), Phys. Rev. Lett. 51, 156 (1983); K. Abe et al. (SHFP collaboration), Phys. Rev. D 33, 1 (1986); M.I. Adamovich, Phys. Lett. B 187, 437 (1987); J.C. Anjos et al. (The Tagged Photon Spectrometer collaboration), Phys. Rev. Lett. 65, 2503 (1990); J.J. Aubert et al. (European Muon Collaboration), Phys. Lett. B 106, 419 (1981)

33. N. Armesto, M.A. Braun, Eur. Phys. J. C 22, 351 (2001)

34. S.J. Brodsky, A.H. Mueller, Phys. Lett. B 206, 685 (1988)

35. M. Genovese, N.N. Nikolaev, B.G. Zakharov, Phys. Lett. B 378, 347 (1996)

36. E.M. Levin, A.D. Martin, M.G. Ryskin, T. Teubner, Z. Phys. C 74, 671 (1997)

37. M. Diehl, Eur. Phys. J. C 1, 293 (1998)

38. A.H. Mueller, Eur. Phys. J. A 1, 19 (1998)

39. N.N. Nikolaev, B.G. Zakharov, V.R. Zoller, Z. Phys. A 351, 435 (1995)

40. M. Arneodo et al., in Proceedings of the workshop on Future Physics at HERA, edited by G. Ingelman, A. De Roeck, R. Klanner, vol. 2, pp. 887-926 (1996) 